\title{
Biconic aggregation functions with a given diagonal or opposite diagonal section
}

\author{
Tarad Jwaid ${ }^{1}$ Bernard De Baets $^{1}$ Hans De Meyer ${ }^{2}$ \\ ${ }^{1}$ Dept. of Applied Mathematics, Biometrics and Process Control, Ghent University \\ Coupure links 653, B-9000 Gent, Belgium \\ ${ }^{2}$ Dept. of Applied Mathematics, Computer Science, Ghent University \\ Krijgslaan 281 S9, B-9000 Gent, Belgium
}

\begin{abstract}
A new method to construct aggregation functions is introduced. These aggregation functions are called biconic aggregation functions with a given diagonal (resp. opposite diagonal) section and their construction method is based on linear interpolation on segments connecting the diagonal (resp. opposite diagonal) of the unit square and the points $(0,1)$ and $(1,0)$ (resp. $(0,0)$ and $(1,1))$. Special classes of biconic aggregation functions such as biconic semicopulas, quasi-copulas and copulas are studied in detail.
\end{abstract}

Keywords: Aggregation function, Quasi-copula, Copula, Diagonal section, Opposite diagonal section, Linear interpolation

\section{Introduction}

A binary aggregation function $A$ is a $[0,1]^{2} \rightarrow[0,1]$ function satisfying the following conditions:

(i) $A(0,0)=0$ and $A(1,1)=1$;

(ii) for any $x, x^{\prime}, y, y^{\prime} \in[0,1]$ such that $x \leq x^{\prime}$ and $y \leq y^{\prime}$, it holds that $A(x, y) \leq A\left(x^{\prime}, y^{\prime}\right)$.

Aggregation functions are of great importance in many fields of application. Their most prominent uses is as logical connectives in fuzzy set theory [2]. To increase modelling flexibility, new methods to construct aggregation functions are being proposed continuously in the literature $[6,18]$.

Special classes of aggregation functions are of particular interest, such as semi-copulas $[15,16]$, triangular norms [1, 22], quasi-copulas [17, 24] and copulas $[1,25]$. They are all conjunctors, in the sense that they extend the classical Boolean conjunction.

Recall that an aggregation function is a semicopula if it has 1 as neutral element, i.e. $A(x, 1)=$ $A(1, x)=x$ for any $x \in[0,1]$. Evidently, any semi-copula $S$ has 0 as annihilator, i.e. $S(0, x)=$ $S(x, 0)=0$ for any $x \in[0,1]$. A semi-copula $S$ is a triangular norm (t-norm for short) if it is commutative and associative. The aggregation functions $T_{\mathbf{M}}$ and $T_{\mathbf{D}}$ given by $T_{\mathbf{M}}(x, y)=\min (x, y)$ and $T_{\mathbf{D}}(x, y)=\min (x, y)$ whenever $\max (x, y)=1$, and $T_{\mathbf{D}}(x, y)=0$ elsewhere, are examples of $\mathrm{t}$ norms. Moreover, for any semi-copula $S$ the inequality $T_{\mathbf{D}} \leq S \leq T_{\mathbf{M}}$ holds. A semi-copula $S$ is a quasi-copula if it is 1-Lipschitz continuous, i.e. for any $x, x^{\prime}, y, y^{\prime} \in[0,1]$ such that $x \leq x^{\prime}$ and $y \leq y^{\prime}$, it holds that

$$
\left|S\left(x^{\prime}, y^{\prime}\right)-S(x, y)\right| \leq\left|x^{\prime}-x\right|+\left|y^{\prime}-y\right| .
$$

A semi-copula $S$ is a copula if it is 2-increasing, i.e. for any $x, x^{\prime}, y, y^{\prime} \in[0,1]$ such that $x \leq x^{\prime}$ and $y \leq y^{\prime}$, it holds that $V_{S}\left(\left[x, x^{\prime}\right] \times\left[y, y^{\prime}\right]\right):=$

$$
S\left(x^{\prime}, y^{\prime}\right)+S(x, y)-S\left(x^{\prime}, y\right)-S\left(x, y^{\prime}\right) \geq 0 .
$$

$V_{S}$ is called the volume of the rectangle $\left[x, x^{\prime}\right] \times$ $\left[y, y^{\prime}\right]$. Any copula is a quasi-copula since the 2-increasingness of a semi-copula implies its 1Lipschitz continuity. The (quasi-)copulas $T_{\mathbf{M}}$ and $T_{\mathbf{L}}$ with $T_{\mathbf{L}}(x, y)=\max (x+y-1,0)$, are respectively the greatest and the smallest (quasi-)copulas, i.e. for any (quasi-)copula $C$, it holds that $T_{\mathbf{L}} \leq$ $C \leq T_{\mathrm{M}}$.

The diagonal section of a $[0,1]^{2} \rightarrow[0,1]$ function $F$ is the function $\delta_{F}:[0,1] \rightarrow[0,1]$ defined by $\delta_{F}(x)=F(x, x)$. A diagonal function [13] is a function $\delta:[0,1] \rightarrow[0,1]$ satisfying the following conditions:

(D1) $\delta(0)=0, \delta(1)=1$;

(D2) $\delta$ is increasing;

(D3) for all $x \in[0,1]$, it holds that $\delta(x) \leq x$;

(D4) $\delta$ is 2-Lipschitz continuous, i.e. for all $x, x^{\prime} \in$ $[0,1]$ it holds that

$$
\left|\delta\left(x^{\prime}\right)-\delta(x)\right| \leq 2\left|x^{\prime}-x\right| .
$$

The set of all diagonal functions will be denoted by $\mathcal{D}$. The set of all $[0,1] \rightarrow[0,1]$ functions that satisfy conditions D1 and D2 (resp. D1, D2 and D3) will be denoted by $\mathcal{D}_{A}\left(\operatorname{resp} . \mathcal{D}_{S}\right)$. The diagonal section of a copula $C$ is a diagonal function. Conversely, for any diagonal function $\delta$, there exists at least one copula $C$ with diagonal section $\delta_{C}=\delta$. For instance, the copula

$$
C_{\delta}(x, y)=\min \left(x, y, \frac{\delta(x)+\delta(y)}{2}\right)
$$


is the greatest symmetric copula with diagonal section $\delta[12,14,26]$. Similarly, the opposite diagonal section of a $[0,1]^{2} \rightarrow[0,1]$ function $F$ is the function $\omega_{F}:[0,1] \rightarrow[0,1]$ defined by $\omega_{F}(x)=F(x, 1-x)$. An opposite diagonal function [7] is a function $\omega$ : $[0,1] \rightarrow[0,1]$ satisfying the following conditions:

(OD1) for all $x \in[0,1]$, it holds that $\omega(x) \leq$ $\min (x, 1-x)$

(OD2) $\omega$ is 1-Lipschitz continuous, i.e. for all $x, x^{\prime} \in[0,1]$, it holds that

$$
\left|\omega\left(x^{\prime}\right)-\omega(x)\right| \leq\left|x^{\prime}-x\right| .
$$

The set of all opposite diagonal functions will be denoted by $\mathcal{O}$. The set of all $[0,1] \rightarrow[0,1]$ functions that satisfy condition OD1 will be denoted by $\mathcal{O}_{S}$. The opposite diagonal section $\omega_{C}$ of a copula $C$ is an opposite diagonal function. Conversely, for any opposite diagonal function $\omega$, there exists at least one copula $C$ with opposite diagonal section $\omega_{C}=\omega$. For instance, the copula $F_{\omega}$ defined by $F_{\omega}(x, y)=T_{\mathbf{L}}(x, y)+$ $\min \{\omega(t) \mid t \in[\min (x, 1-y), \max (x, 1-y)]\} \quad$ is the greatest copula with opposite diagonal section $\omega[7,23]$. Note that $F_{\omega}$ is opposite symmetric [7], i.e. $F_{\omega}(x, y)-F_{\omega}(1-y, 1-x)=x+y-1$, for any $(x, y) \in[0,1]^{2}$. Diagonal and opposite diagonal functions have been used recently to construct several subclasses of aggregation functions such as quasi-copulas and copulas [5, 7, 11, 12, 13, 14, 20].

Characteristic for the aggregation function $T_{\mathbf{M}}$ is that its surface is constituted from linear segments connecting its diagonal section to the points $(0,1,0)$ and $(1,0,0)$. Also characteristic for $T_{\mathbf{M}}$ is that its surface is constituted from linear segments connecting its opposite diagonal section to the points $(0,0,0)$ and $(1,1,1)$. Inspired by the above interpretation, we introduce a new method to construct aggregation functions. These aggregation functions are constructed by linear interpolation on segments connecting the diagonal (resp. opposite diagonal) of the unit square to the points $(0,1)$ and $(1,0)$ (resp. $(0,0)$ and $(1,1))$.

This paper is organized as follows. In the next section we introduce the definition of a biconic function with a given diagonal section and characterize the class of biconic aggregation functions, as well as the classes of biconic semi-copulas, biconic quasicopulas, biconic copulas and singular biconic copulas. The class of biconic functions with a given opposite diagonal section is introduced in Section 3. Finally, some conclusions are given.

\section{Biconic functions with a given diagonal section}

\subsection{Biconic aggregation functions with a given diagonal section}

In this subsection we introduce biconic functions with a given diagonal section. Their construction is based on linear interpolation on segments connecting the diagonal of the unit square and the points $(0,1)$ and $(1,0)$. Throughout this paper the convention $\frac{0}{0}=0$ is assumed. For any $(x, y) \in[0,1]^{2}$, we introduce in this subsection the following notations

$$
u=\frac{x}{1+x-y}, \quad v=\frac{y}{1+y-x} .
$$

Let $\delta \in \mathcal{D}_{A}$ and $\alpha, \beta \in[0,1]$. The function $A_{\delta}^{\alpha, \beta}$ : $[0,1]^{2} \rightarrow[0,1]$ defined by

$$
A_{\delta}^{\alpha, \beta}(x, y)= \begin{cases}\alpha(x-y)+y \frac{\delta(v)}{v} & , \text { if } y \leq x \\ \beta(y-x)+x \frac{\delta(u)}{u} & , \text { otherwise }\end{cases}
$$

is well defined. This function is called a biconic function with a given diagonal section.

Proposition 1 Let $\delta \in \mathcal{D}_{A}$. The function $A_{\delta}^{\alpha, \beta}$ defined in (1) is an aggregation function if and only if

(i) the functions $\left.\left.\mu_{\delta, \alpha}, \mu_{\delta, \beta}:\right] 0,1\right] \rightarrow \mathbb{R}$, defined by

$$
\mu_{\delta, \alpha}(x)=\frac{\delta(x)-\alpha}{x}, \quad \mu_{\delta, \beta}(x)=\frac{\delta(x)-\beta}{x},
$$

are increasing;

(ii) the functions $\lambda_{\delta, \alpha}, \lambda_{\delta, \beta}:[0,1[\rightarrow \mathbb{R}$, defined by

$$
\lambda_{\delta, \alpha}(x)=\frac{\delta(x)-\alpha}{1-x}, \quad \lambda_{\delta, \beta}(x)=\frac{\delta(x)-\beta}{1-x},
$$

are increasing.

Inspired by the above proposition, the biconic function $A_{\delta}^{\alpha, \beta}$ is called a biconic aggregation function with a given diagonal section.

Example 1 Consider the diagonal section $\delta_{\mathbf{M}}$ of $T_{\mathbf{M}}$, i.e. $\delta_{\mathbf{M}}(x)=x$ for any $x \in[0,1]$. Obviously, conditions ( $i$ ) and (ii) of Proposition 1 are satisfied. The resulting biconic aggregation function is a Choquet integral $[4,10]$, i.e.

$$
A_{\delta_{\mathrm{M}}}^{\alpha, \beta}(x, y)= \begin{cases}\alpha x+(1-\alpha) y & , \text { if } y \leq x, \\ (1-\beta) x+\beta y & , \text { otherwise. }\end{cases}
$$

Taking $\beta=1-\alpha$, the resulting biconic aggregation function is the weighed arithmetic mean, i.e.

$$
A_{\delta_{\mathrm{M}}}^{\alpha, 1-\alpha}(x, y)=\alpha x+(1-\alpha) y .
$$

Proposition 2 Let $A_{\delta}^{\alpha, \beta}$ be a biconic aggregation function. Then the inequality

$\max (\alpha x, \beta x) \leq \delta(x) \leq \min (\alpha+(1-\alpha) x, \beta+(1-\beta) x)$,

holds for any $x \in[0,1]$. 
Now we identify the functions in $\mathcal{D}_{A}$ which characterize the extreme biconic aggregation functions with fixed $\alpha$ and $\beta$. Let $\alpha, \beta \in[0,1]$ and consider the functions $\underline{\delta}^{\alpha, \beta}, \bar{\delta}^{\alpha, \beta}:[0,1] \rightarrow[0,1]$, defined by

$$
\begin{aligned}
& \underline{\delta}^{\alpha, \beta}(x)= \begin{cases}\max (\alpha x, \beta x) & , \text { if } x<1, \\
1 & , \text { if } x=1,\end{cases} \\
& \bar{\delta}^{\alpha, \beta}(x)= \begin{cases}\min (\alpha+(1-\alpha) x, \beta+(1-\beta) x) \\
& , \text { if } x>0, \\
0 & , \text { if } x=0 .\end{cases}
\end{aligned}
$$

Obviously, $\underline{\delta}^{\alpha, \beta}, \bar{\delta}^{\alpha, \beta} \in \mathcal{D}_{A}$ and the conditions of Proposition 1 are satisfied. Note also that for any two biconic aggregation functions $A_{\delta_{1}}^{\alpha, \beta}$ and $A_{\delta_{2}}^{\alpha, \beta}$, it holds that $A_{\delta_{1}}^{\alpha, \beta} \leq A_{\delta_{2}}^{\alpha, \beta}$ if and only if $\delta_{1} \leq \delta_{2}$. The following proposition is then obvious.

Proposition 3 Let $A_{\delta}^{\alpha, \beta}$ be a biconic aggregation function. Then it holds that

$$
A_{\underline{\delta}^{\alpha, \beta}}^{\alpha, \beta} \leq A_{\delta}^{\alpha, \beta} \leq A_{\bar{\delta}^{\alpha, \beta}}^{\alpha, \beta} .
$$

Example 2 The functions $\underline{\delta}^{0,0}$ and $\bar{\delta}^{0,0}$ are given by

$$
\underline{\delta}^{0,0}(x)= \begin{cases}0 \quad & \text { if } x<1, \\ 1 \quad, \text { if } x=1,\end{cases}
$$

and $\bar{\delta}^{0,0}(x)=x$ for any $x \in[0,1]$. The corresponding biconic aggregation functions are respectively the smallest $t$-norm, i.e. $A_{\underline{\delta}^{0,0}}^{0,0}=T_{\mathbf{D}}$, and the greatest t-norm, i.e. $A_{\bar{\delta}^{0,0}}^{0,0}=T_{\mathbf{M}}$.

Example 3 The functions $\underline{\delta}^{1,1}$ and $\bar{\delta}^{1,1}$ are given by $\underline{\delta}^{1,1}(x)=x$ for any $x \in[0,1]$ and

$$
\bar{\delta}^{1,1}(x)= \begin{cases}1 & , \text { if } x>0 \\ 0 & , \text { if } x=0\end{cases}
$$

The corresponding biconic aggregation functions are respectively the smallest t-conorm, i.e. $A_{\underline{\delta}^{1,1}}^{1,1}(x, y)=\max (x, y)$, and the greatest $t$-conorm, i.e. $A_{\bar{\delta}^{1,1}}^{1,1}(x, y)=\max (x, y)$ whenever $\min (x, y)=0$, and $A_{\bar{\delta}^{1,1}}^{1,1}(x, y)=1$ elsewhere.

Example 4 The functions $\underline{\delta}^{1,0}, \bar{\delta}^{1,0}, \underline{\delta}^{0,1}$ and $\bar{\delta}^{0,1}$ all coincide with $\delta_{\mathbf{M}}$. The corresponding biconic aggregation functions coincide with the projection to the first and second coordinate $[2,3]$, i.e. $A_{\underline{\delta}^{1,0}}^{1,0}(x, y)=A_{\bar{\delta}^{1,0}}^{1,0}(x, y)=x$ and $A_{\underline{\delta}^{0,1}}^{0,1}(x, y)=$ $A_{\bar{\delta}^{0,1}}^{\overline{0}, 1}(x, y)=y$.
Evidently, any biconic function $A_{\delta}^{\alpha, \beta}$ is continuous if and only if $\delta$ is continuous. The functions $\underline{\delta}^{\alpha, \beta}$ and $\bar{\delta}^{\alpha, \beta}$ need not to be continuous in general. In fact, the only case in which they are both continuous is when

$$
\max (\alpha, \beta)=1 \text { and } \min (\alpha, \beta)=0 .
$$

However, as Example 4 shows, it then holds that

$$
\underline{\delta}^{\alpha, \beta}=\delta=\bar{\delta}^{\alpha, \beta}=\delta_{\mathbf{M}}
$$

and $A_{\delta}^{\alpha, \beta}$ coincides with one of the projections.

Proposition 4 Let $\delta \in \mathcal{D}_{A}$. The function $A_{\delta}^{\alpha, \beta}$ defined in (1)

(i) is symmetric, i.e. $A_{\delta}^{\alpha, \beta}(x, y)=A_{\delta}^{\alpha, \beta}(y, x)$ for any $(x, y) \in[0,1]^{2}$ if and only if $\alpha=\beta$;

(ii) has 0 as annihilator if and only if $\alpha=\beta=0$;

(iii) has 1 as neutral element if and only if $\alpha=$ $\beta=0$.

From here on, we will only consider biconic functions with a given diagonal section that have 1 as neutral element, i.e. $\alpha=\beta=0$. We then abbreviate $A_{\delta}^{0,0}$ as $A_{\delta}$. In this case, $A_{\delta}$ is symmetric and is given by

$$
A_{\delta}(x, y)= \begin{cases}y \frac{\delta(v)}{v} & , \text { if } y \leq x \\ x \frac{\delta(u)}{u} & , \text { otherwise }\end{cases}
$$

Suppose that the diagonal section of a biconic aggregation function $A_{\delta}$ contains a (linear) segment with endpoints $\left(x_{1}, \delta\left(x_{1}\right)\right)$ and $\left(x_{2}, \delta\left(x_{2}\right)\right)$. From the definition of a biconic function with a given diagonal section, it follows that $A_{\delta}$ is linear on the triangle $T_{1}:=\Delta_{\left\{\left(x_{1}, x_{1}\right),\left(x_{2}, x_{2}\right),(1,0)\right\}}$ as well as on the triangle $T_{2}:=\Delta_{\left\{\left(x_{1}, x_{1}\right),\left(x_{2}, x_{2}\right),(0,1)\right\}}$. This situation is depicted in Figure 1. For any $(x, y) \in T_{1}$, it

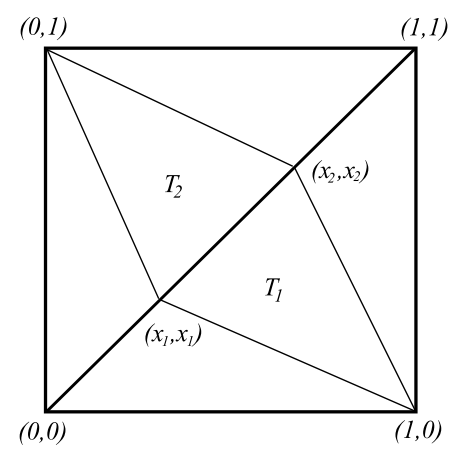

Figure 1: Illustration for the triangles $T_{1}$ and $T_{2}$.

holds that

$$
A_{\delta}(x, y)=a x+b y+c .
$$


Furthermore,

$$
\begin{aligned}
a x_{1}+b x_{1}+c & =\delta\left(x_{1}\right) \\
a x_{2}+b x_{2}+c & =\delta\left(x_{2}\right) \\
a+c & =0 .
\end{aligned}
$$

Solving this system of equations and using the symmetry of $A_{\delta}$, we obtain

$$
A_{\delta}(x, y)= \begin{cases}\frac{r x+s y-r}{t} & , \text { if }(x, y) \in T_{1}, \\ \frac{s x+r y-r}{t} & , \text { if }(x, y) \in T_{2},\end{cases}
$$

where

$$
\begin{aligned}
r & =x_{1} \delta\left(x_{2}\right)-x_{2} \delta\left(x_{1}\right) \\
s & =\left(1-x_{1}\right) \delta\left(x_{2}\right)-\left(1-x_{2}\right) \delta\left(x_{1}\right) \\
t & =x_{2}-x_{1} .
\end{aligned}
$$

\subsection{Biconic semi-copulas with a given diagonal section}

In this subsection we characterize the set of functions in $\mathcal{D}_{S}$ for which the corresponding biconic function is a semi-copula.

Lemma 1 Let $\delta \in \mathcal{D}_{S}$. The function $\lambda_{\delta}:[0,1[\rightarrow$ $\left[0, \infty\left[\right.\right.$, defined by $\lambda_{\delta}(x)=\frac{\delta(x)}{1-x}$, is increasing;

Proposition 5 Let $\delta \in \mathcal{D}_{S}$. Then the function $A_{\delta}:[0,1]^{2} \rightarrow[0,1]$ defined in (3) is a semi-copula if and only if the function $\left.\left.\mu_{\delta}:\right] 0,1\right] \rightarrow[0, \infty[$, defined by $\mu_{\delta}(x)=\frac{\delta(x)}{x}$, is increasing.

Example 5 Consider the diagonal functions $\delta_{\mathbf{M}}$ and $\delta_{\mathbf{L}}$ with $\delta_{\mathbf{L}}$ being the diagonal section of $T_{\mathbf{L}}$, i.e. $\delta_{\mathbf{L}}(x)=\max (2 x-1,0)$ for any $x \in[0,1]$. Clearly, the functions $\mu_{\delta_{\mathbf{M}}}$ and $\mu_{\delta_{\mathbf{L}}}$, defined in Proposition 5, are increasing. The corresponding biconic semicopulas are respectively $T_{\mathbf{M}}$ and $T_{\mathbf{L}}$.

Example 6 Consider the diagonal function $\delta(x)=$ $x^{1+\theta}$ with $\theta \in[0,1]$. Clearly, the function $\mu_{\delta}$, defined in Proposition 5, is increasing for any $\theta \in$ $[0,1]$. The corresponding family of biconic semicopulas is given by

$$
A_{\theta}(x, y)= \begin{cases}\frac{y^{1+\theta}}{(1+y-x)^{\theta}} & , \text { if } y \leq x \\ \frac{x^{1+\theta}}{(1+x-y)^{\theta}} & , \text { otherwise. }\end{cases}
$$

Proposition 6 Let $A_{\delta}$ be a biconic semi-copula and suppose that $\delta\left(x_{0}\right)=x_{0}$ for some $\left.x_{0} \in\right] 0,1[$. Then it holds that $\delta(x)=x$ for any $x \in\left[x_{0}, 1\right]$.

\subsection{Biconic quasi-copulas with a given diagonal section}

An interesting class of aggregation functions is the class of quasi-copulas. Quasi-copulas are of increasing importance in various studies in fuzzy set theory, such as preference modelling and similarity measurement $[8,9]$.

Next we characterize the diagonal functions for which the corresponding biconic function is a quasicopula.

Lemma 2 Let $\delta \in \mathcal{D}$. Then it holds that

(i) the function $\left.\left.\nu_{\delta}:\right] 0,1\right] \rightarrow[2, \infty[$, defined by $\nu_{\delta}(x)=\frac{1+\delta(x)}{x}$, is decreasing;

(ii) the function $\phi_{\delta}:[0,1 / 2[\cup] 1 / 2,1] \rightarrow \mathbb{R}$, defined by $\phi_{\delta}(x)=\frac{\delta(x)}{1-2 x}$, is increasing on the interval $[0,1 / 2[$ and on the interval $] 1 / 2,1]$.

Proposition 7 Let $\delta \in \mathcal{D}$. Then the function $A_{\delta}$ : $[0,1]^{2} \rightarrow[0,1]$ defined in (3) is a quasi-copula if and only if

(i) the function $\mu_{\delta}$, defined in Proposition 5, is increasing;

(ii) the function $\xi_{\delta}:[0,1[\rightarrow[0,1[$, defined by $\xi_{\delta}(x)=\frac{x-\delta(x)}{1-x}$, is increasing.

Example 7 Consider the diagonal function in Example 6. Clearly, the functions $\mu_{\delta}$ and $\xi_{\delta}$, defined in Propositions 5 and 7 , are increasing. The corresponding family of biconic semi-copulas is a family of biconic quasi-copulas.

Example 8 Consider the diagonal function $\delta$ defined by

$$
\delta(x)= \begin{cases}0 & , \text { if } x \leq \frac{1}{6}, \\ 2 x-\frac{1}{3} & , \text { if } \frac{1}{6} \leq x \leq \frac{1}{4}, \\ \frac{2}{3} x & , \text { if } \frac{1}{4} \leq x \leq \frac{3}{4}, \\ 2 x-1 & , \text { otherwise. }\end{cases}
$$

Clearly, the function $\mu_{\delta}$, defined in Proposition 5, $i s$ increasing. Note also that the function $\xi_{\delta}$, defined in Proposition 7, is not increasing. Hence, the corresponding biconic semi-copula is a proper semicopula. Consequently, the class of biconic quasicopulas with a given diagonal section is a proper subclass of the class of biconic semi-copulas with a given diagonal section.

Proposition 8 Let $A_{\delta}$ be a biconic quasi-copula. Then it holds that

(i) if $\delta\left(x_{0}\right)=x_{0}$ for some $\left.x_{0} \in\right] 0,1\left[\right.$, then $A_{\delta}=$ $T_{\mathrm{M}}$

(ii) if $\delta\left(x_{0}\right)=2 x_{0}-1$ for some $x_{0} \in[1 / 2,1[$, then $\delta(x)=2 x-1$ for any $x \in\left[x_{0}, 1\right]$. 

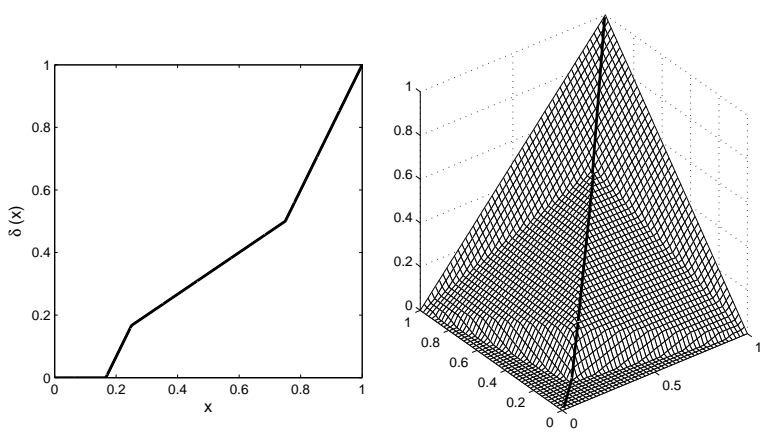

Figure 2: The diagonal function and the corresponding biconic semi-copula of Example 8 .

\subsection{Biconic copulas with a given diagonal section}

Another relevant class of aggregation functions is the class of copulas. Due to Sklar's theorem [27], copulas have received increasing attention from researchers in statistics and probability theory [19]. We denote the (linear) segment with endpoints $\mathbf{x}, \mathbf{y} \in[0,1]^{2}$ as

$$
\langle\mathbf{x}, \mathbf{y}\rangle=\{\theta \mathbf{x}+(1-\theta) \mathbf{y} \mid \theta \in[0,1]\} .
$$

Proposition 9 Let $\delta$ be a piecewise linear diagonal function. Then the function $A_{\delta}:[0,1]^{2} \rightarrow[0,1]$ defined in (3) is a copula if and only if $\delta$ is convex.

Lemma 3 Let $C_{\delta}$ be a biconic copula and $m_{1}, m_{2} \in$ ] $-\infty, 0\left[\right.$ such that $m_{1}>m_{2}$. Consider three points $\mathbf{b}_{1}:=\left(x_{1}, x_{1}\right), \mathbf{b}_{2}:=\left(x_{2}, x_{2}\right)$ and $\mathbf{b}_{3}:=\left(x_{3}, x_{3}\right)$ such that $0 \leq x_{1}<x_{2}<x_{3} \leq 1$ and the segments $\left\langle\mathbf{b}_{1},(1,0)\right\rangle,\left\langle\mathbf{b}_{2},(1,0)\right\rangle$ and $\left\langle\mathbf{b}_{3},(1,0)\right\rangle$ have slopes $m_{1},-\sqrt{m_{1} m_{2}}$ and $m_{2}$, respectively. Then it holds that

(i) there exists a rectangle $\left[x, x^{\prime}\right] \times\left[y, y^{\prime}\right]$ such that the segment connecting the points $\left(x, y^{\prime}\right)$ and $\left(x^{\prime}, y\right)$ is a subset of the segment $\left\langle\mathbf{b}_{2},(1,0)\right\rangle$ and the points $(x, y)$ and $\left(x^{\prime}, y^{\prime}\right)$ are located on the segments $\left\langle\mathbf{b}_{1},(1,0)\right\rangle$ and $\left\langle\mathbf{b}_{3},(1,0)\right\rangle$ respectively.

(ii) the point $\left(x_{2}, \delta\left(x_{2}\right)\right)$ lies below or on the segment $\left\langle\left(x_{1}, \delta\left(x_{1}\right)\right),\left(x_{3}, \delta\left(x_{3}\right)\right)\right\rangle$.

Lemma 3 and Proposition 9 are used to show that for any convex diagonal function, the function $A_{\delta}$ defined in (3) is a copula.

Proposition 10 Let $\delta \in \mathcal{D}$. Then the function $A_{\delta}:[0,1]^{2} \rightarrow[0,1]$ defined in (3) is a copula if and only if $\delta$ is convex.

Example 9 Consider the diagonal function in Example 6. Clearly, $\delta$ is convex for any $\theta \in[0,1]$. The corresponding family of biconic semi-copulas is a family of biconic copulas.
Example 10 Consider the diagonal function given by $\delta(x)=\frac{x^{2}}{1-\theta(1-x)^{2}}$ with $\theta \in[-1,1]$. Clearly, $\delta$ is convex for any $\theta \in[-1,1]$. The corresponding family of biconic copulas is given by

$C_{\theta}(x, y)= \begin{cases}\frac{y^{2}(1+y-x)}{(1+y-x)^{2}-\theta(1-x)^{2}} \quad, \text { if } y \leq x, \\ \frac{x^{2}(1+x-y)}{(1+x-y)^{2}-\theta(1-y)^{2}} \quad, \text { otherwise. }\end{cases}$

Example 11 Consider the diagonal function $\delta$ given by

$$
\delta(x)= \begin{cases}0 & , \text { if } x \leq \frac{1}{4}, \\ \frac{1}{3}(4 x-1) & , \text { if } \frac{1}{4} \leq x \leq \frac{2}{5}, \\ \frac{1}{2} x & , \text { if } \frac{2}{5} \leq x \leq \frac{2}{3}, \\ 2 x-1 & , \text { otherwise. }\end{cases}
$$

Clearly, the functions $\mu_{\delta}$ and $\xi_{\delta}$, defined in Propositions 5 and 7 , are increasing. Note also that $\delta$ is not convex. Hence, $A_{\delta}$ is a proper biconic quasicopula. Consequently, the class of biconic copulas
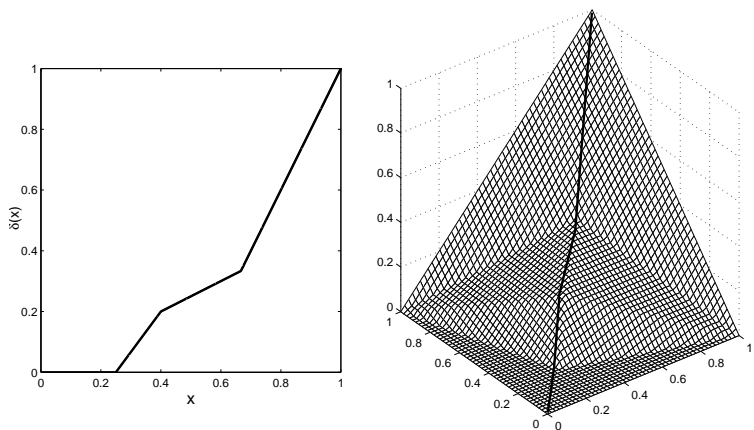

Figure 3: The diagonal function and the corresponding biconic quasi-copula of Example 11.

with a given diagonal section is a proper subclass of the class of biconic quasi-copulas with a given diagonal section.

In the following lemma the opposite symmetry property of a biconic copula with a given diagonal section is studied.

Proposition $11 \mathrm{~A}$ biconic copula $C_{\delta}$ is opposite symmetric if and only if the function $f(x)=x-\delta(x)$ is symmetric with respect to the point $(1 / 2,1 / 2)$, i.e. $\delta(x)-\delta(1-x)=2 x-1$ for any $x \in[0,1 / 2]$.

Next we characterize the class of singular biconic copulas with a given diagonal section. The support of a copula $C$ is the complement of the union of all non-degenerated open rectangles of the unit square such that the $C$-volume of the closed rectangle is equal to zero. A copula $C$ is called singular if its support has Lebesgue measure zero. 
Proposition 12 Let $C_{\delta}$ be a biconic copula. Then it holds that $C_{\delta}$ is singular if and only if $\delta$ is piecewise linear.

Example 12 The family of biconic copulas given in (7) is a family of singular biconic copulas.

We focus now on associative biconic copulas with a given diagonal section and conclude that the only two associative biconic copulas with a given diagonal section are $T_{\mathbf{M}}$ and $T_{\mathbf{L}}$. Every 1-Lipschitz tnorm is a copula, while every associative copula is a t-norm (the commutativity can be obtained from the continuity of a copula [22]).

Proposition $13 T_{\mathbf{M}}$ and $T_{\mathbf{L}}$ are the only biconic associative copulas (1-Lipschitz t-norms) with a given diagonal section.

We conclude this subsection by finding the intersection between the set of biconic copulas with a given diagonal section and the set of conic copulas. Conic copulas were introduced in [21] and their construction was based on linear interpolation on segments connecting the upper boundary curve of the zero-set and the point $(1,1)$. In other words, the surface of any conic copula is constituted from its zero-set and segments connecting the upper boundary curve of its zero-set to the point $(1,1,1)$. The zero-set $Z_{C}$ of a copula $C$ is the inverse image of the value 0 , i.e.

$Z_{C}:=C^{-1}(\{0\})=\left\{(x, y) \in[0,1]^{2} \mid C(x, y)=0\right\}$.

Lemma 4 Let $\delta \in \mathcal{D}$ and suppose that $\theta=1-$ $2^{-\frac{1}{\theta_{1}}}$, with $\theta_{1} \in[1, \infty[$, is the maximum value such that $\delta(\theta)=0$. Then the biconic copula $C_{\delta}$ has the zero set $Z_{C_{\delta}}$ given by

$$
Z_{C_{\delta}}=\left\{(x, y) \in[0,1]^{2} \mid y \leq f_{\theta_{1}}(x)\right\}
$$

where the function $f_{\theta_{1}}:[0,1] \rightarrow[0,1]$ is given by

$$
f_{\theta_{1}}(x)= \begin{cases}\left(1-2^{\frac{1}{\theta_{1}}}\right)^{-1} x+1 & , \text { if } x \leq 1-2^{-\frac{1}{\theta_{1}}}, \\ \left(1-2^{\frac{1}{\theta_{1}}}\right)(x-1) & \text { if } x \geq 1-2^{-\frac{1}{\theta_{1}}} .\end{cases}
$$

Due to the above lemma and the definition of a conic copula, the following proposition is obvious.

Proposition 14 Let $C_{\delta}$ be a biconic copula and suppose further that $C_{\delta}$ is a conic copula. Then it holds that

$$
\begin{aligned}
& C_{\delta}(x, y)= \\
& \left\{\begin{array}{l}
\max \left(y+(1-x)\left(1-2^{\frac{1}{\theta_{1}}}\right), 0\right) \quad, \text { if } y \leq x, \\
\max \left(x+(1-y)\left(1-2^{\frac{1}{\theta_{1}}}\right), 0\right), \text { otherwise, }
\end{array}\right.
\end{aligned}
$$

with $\theta_{1} \in[1, \infty[$. This family of copulas was introduced in [21].

\section{Biconic functions with a given opposite diagonal section}

In this section we introduce biconic functions with a given opposite diagonal section. Their construction is based on linear interpolation on segments connecting the opposite diagonal of the unit square and the points $(0,0)$ and $(1,1)$. For any $(x, y) \in[0,1]^{2}$, we introduce in this section the following notations

$$
u^{\prime}=\frac{x}{x+y}, \quad v^{\prime}=\frac{1-y}{2-x-y} .
$$

Let $\omega:[0,1] \rightarrow[0,1]$ and $\alpha, \beta \in[0,1]$. The function $A_{\omega}^{\alpha, \beta}:[0,1]^{2} \rightarrow[0,1]$ defined by $A_{\omega}^{\alpha, \beta}(x, y)=$

$$
\begin{cases}\alpha(1-x-y)+x \frac{\omega\left(u^{\prime}\right)}{u^{\prime}} & , \text { if } x+y \leq 1, \\ \beta(x+y-1)+(1-y) \frac{\omega\left(v^{\prime}\right)}{v^{\prime}} & , \text { otherwise. }\end{cases}
$$

is well defined. This function is called a biconic function with a given opposite diagonal section. Evidently, the boundary conditions of an aggregation function imply that $\alpha=0$ and $\beta=1$. We then abbreviate $A_{\omega}^{0,1}$ as $A_{\omega}$ with $A_{\omega}(x, y)=$

$$
\begin{cases}x \frac{\omega\left(u^{\prime}\right)}{u^{\prime}} & , \text { if } x+y \leq 1 \\ x+y-1+(1-y) \frac{\omega\left(v^{\prime}\right)}{v^{\prime}} & , \text { otherwise }\end{cases}
$$

Clearly, the function $A_{\omega}$ defined in (9) has 1 as neutral element. Therefore, if $A_{\omega}$ is an aggregation function then it is also a semi-copula.

In the next proposition, we characterize the functions in $\mathcal{O}_{S}$ for which the corresponding biconic function is a biconic aggregation function.

Proposition 15 Let $\omega \in \mathcal{O}_{S}$. Then the function $A_{\omega}:[0,1]^{2} \rightarrow[0,1]$ defined in (9) is an aggregation function if and only if

(i) the functions $\left.\left.\mu_{\omega}, \rho_{\omega}:\right] 0,1\right] \rightarrow[0,1]$, defined by $\mu_{\omega}(x)=\frac{\omega(x)}{x}, \rho_{\omega}(x)=\frac{1-\omega(x)}{x}$, are decreasing;

(ii) the functions $\lambda_{\omega}, \xi_{\omega}:[0,1[\rightarrow[0,1]$, defined by $\lambda_{\omega}(x)=\frac{\omega(x)}{1-x}$ and $\xi_{\omega}=\frac{x-\omega(x)}{1-x}$, are increasing.

Let $C$ be a quasi-copula (resp. copula) with opposite diagonal section $\omega$. The function $C^{\prime}$, defined by $C^{\prime}(x, y)=x-C(x, 1-y)$, is again a quasi-copula (resp. copula) whose diagonal section $\delta_{C^{\prime}}$ is given by $\delta_{C^{\prime}}(x)=x-\omega(x)$. This transformation permits to derive in a straightforward manner the conditions that have to be satisfied by an opposite diagonal function to obtain a biconic quasi-copula (resp. copula), which has that opposite diagonal function as opposite diagonal section.

Proposition 16 Let $\omega \in \mathcal{O}$. Then the function $A_{\omega}:[0,1]^{2} \rightarrow[0,1]$ defined in (9) is a quasicopula if and only if the functions $\mu_{\omega}$ and $\lambda_{\omega}$, defined in Proposition 15, are respectively decreasing and increasing. 
Proposition 17 Let $\omega \in \mathcal{O}$. Then the function $A_{\omega}:[0,1]^{2} \rightarrow[0,1]$ defined in (9) is a copula if and only if $\omega$ is concave.

Example 13 Consider the opposite diagonal functions $\omega_{\mathbf{M}}(x)=\min (x, 1-x)$ and $\omega_{\mathbf{L}}(x)=0$. Obviously, $\omega_{\mathbf{M}}$ and $\omega_{\mathbf{L}}$ are concave functions. The corresponding biconic copulas are respectively $T_{\mathbf{M}}$ and $T_{\mathbf{L}}$.

Example 14 Consider the opposite diagonal function $\omega_{\Pi}(x)=x(1-x)$. Obviously, $\omega_{\Pi}$ is concave. The corresponding biconic copula is given by

$$
C_{\omega_{\Pi}}(x, y)= \begin{cases}x\left(1-u^{\prime}\right) & , \text { if } x+y \leq 1 \\ x-(1-y) v^{\prime} & , \text { otherwise }\end{cases}
$$

We focus now on the symmetry and opposite symmetry properties of biconic copulas with a given opposite diagonal section.

Proposition 18 Let $C_{\omega}$ be a biconic copula. Then it holds that

(i) $C_{\omega}$ is opposite symmetric;

(ii) $C_{\omega}$ is symmetric if and only if $\omega$ is symmetric with respect to the point $(1 / 2,1 / 2)$, i.e. $\omega(x)=$ $\omega(1-x)$ for any $x \in[0,1 / 2]$.

We conclude this section by finding the intersection between the class of biconic copulas with a given opposite diagonal section and the class of biconic copulas with a given diagonal section and the class of conic copulas.

Proposition 19 Let $C$ be a biconic copula with a given opposite diagonal section and suppose further that $C$ is a biconic copula with a given diagonal section. Then it holds that $C$ is a member of the following family

$$
\theta T_{\mathbf{M}}+(1-\theta) T_{\mathbf{L}} \quad \text { with } \theta \in[0,1] .
$$

Let $C_{\omega}$ be a biconic copula. Due to the definition of $C_{\omega}$, the only possible zero-sets are

$$
\left.\left.Z_{C_{\omega}}=Z_{T_{\mathrm{M}}}=[0,1]^{2} \backslash\right] 0,1\right]^{2}
$$

and

$$
Z_{C_{\omega}}=Z_{T_{\mathbf{L}}}=\left\{(x, y) \in[0,1]^{2} \mid x+y \leq 1\right\} .
$$

Recalling that every conic copula is uniquely determined by its zero-set [21], the following proposition is clear.

Proposition 20 Let $C_{\omega}$ be a biconic copula with given opposite diagonal section $\omega$ and suppose further that $C_{\omega}$ is a conic copula. Then it holds that $C_{\omega}=T_{\mathbf{M}}$ or $C_{\omega}=T_{\mathbf{L}}$.

\section{Conclusion}

We have introduced biconic aggregation functions with a given diagonal (resp. opposite diagonal) section. We have also characterized the classes of biconic semi-copulas, quasi-copulas and copulas with a given diagonal (resp. opposite diagonal) section. The t-norms $T_{\mathbf{M}}$ and $T_{\mathbf{L}}$ turn out to be the only 1-Lipschitz biconic t-norms with a given diagonal section. Moreover, a copula that is a biconic copula with a given diagonal section as well as with a given opposite diagonal section turns out to be a convex combination of $T_{\mathbf{M}}$ and $T_{\mathbf{L}}$.

\section{References}

[1] C. Alsina, M.J. Frank and B. Schweizer, Associative Functions: Triangular Norms and Copulas, World Scientific, Singapore, 2006.

[2] G. Beliakov, A. Pradera and T. Calvo, Aggregation Functions: a Guide for Practitioners, Studies in Fuzziness and Soft Computing, Vol. 221, Springer, Berlin, 2007.

[3] T. Calvo, A. Kolesárová, M. Komorníková and R. Mesiar, Aggregation Operators: Properties, Classes and Construction Methods. In: Aggregation Operators, New Trends and Applications, Physica-Verlag, New York, 2002, pp. 3106.

[4] G. Choquet, Theory of capacities, Annales Institute Fourier, 5:131-295, 1953.

[5] B. De Baets, H. De Meyer and R. Mesiar, Asymmetric semilinear copulas, Kybernetika 43:221-233, 2007.

[6] B. De Baets, H. De Meyer and R. Mesiar, Piecewise linear aggregation functions based on triangulation, Information Sciences, 181:466478, 2011.

[7] B. De Baets, H. De Meyer and M. ÚbedaFlores, Opposite diagonal sections of quasicopulas and copulas, Internat. J. Uncertainty, Fuzziness and Knowledge-Based Systems, 17:481-490, 2009.

[8] B. De Baets and J. Fodor, Additive fuzzy preference structures: the next generation, in: Principles of Fuzzy Preference Modelling and Decision Making (B. De Baets and J. Fodor, eds.), Academia Press, 2003, pp. 15-25.

[9] B. De Baets, S. Janssens and H. De Meyer, On the transitivity of a parametric family of cardinality-based similarity measures, Internat. J. Approximate Reasoning, 50:104-116, 2009.

[10] D. Denneberg, Non-additive Measure and Integral, Kluwer, 1994.

[11] F. Durante and P. Jaworski, Absolutely continuous copulas with given diagonal sections, Communications in Statistics: Theory and Methods, 37:2924-2942, 2008. 
[12] F. Durante, A. Kolesárová, R. Mesiar and C. Sempi, Copulas with given diagonal sections, novel constructions and applications, Internat. J. Uncertainty, Fuzziness and KnowledgeBased Systems, 15:397-410, 2007.

[13] F. Durante, A. Kolesárová, R. Mesiar and C. Sempi, Semilinear copulas, Fuzzy Sets and Systems, 159:63-76, 2008.

[14] F. Durante, R. Mesiar and C. Sempi, On a family of copulas constructed from the diagonal section, Soft Computing, 10:490-494, 2006.

[15] F. Durante, J.J. Quesada Molina and C. Sempi, Semicopulas: characterizations and applicability, Kybernetika, 42:287-302, 2006.

[16] F. Durante and C. Sempi, Semicopulae, Kybernetika, 41:315-328, 2005.

[17] C. Genest, J.J. Quesada Molina, J.A. Rodríguez Lallena and C. Sempi, A characterization of quasi-copulas, Journal of Multivariate Analysis, 69:193-205, 1999.

[18] M. Grabisch, J.-L. Marichal, R. Mesiar and E. Pap, Aggregation functions: construction methods, conjunctive, disjunctive and mixed classes, Information Sciences, 181:23-43, 2011.

[19] H. Joe, Multivariate Models and Dependence Concepts, Chapman \& Hall, London, 1997.

[20] T. Jwaid, B. De Baets and H. De Meyer, Orbital semilinear copulas, Kybernetika 45:10121029, 2009.

[21] T. Jwaid, B. De Baets, J. Kalická and R. Mesiar, Conic aggregation functions, Fuzzy Sets and Systems 167:3-20, 2011.

[22] E.P. Klement, R. Mesiar and E. Pap, Triangular Norms, Trends in Logic, Studia Logica Library, Vol. 8, Kluwer Academic Publishers, Dordrecht, 2000.

[23] E.P. Klement and A. Kolesárová, Extension to copulas and quasi-copulas as special 1Lipschitz aggregation operators, Kybernetika, 41:329-348, 2005.

[24] A. Kolesárová, 1-Lipschitz aggregation operators and quasi-copulas, Kybernetika, 39:615629, 2003.

[25] R. Nelsen, An Introduction to Copulas, Springer, New York, 2006.

[26] R. Nelsen and G. Fredricks, Diagonal copulas. In: V. Beneš and J. Štěpán, Eds., Distributions with given Marginals and Moment Problems, Kluwer Academic Publishers, Dordrecht, 1977, pp. $121-127$.

[27] A. Sklar, Fonctions de répartition à $n$ dimensions et leurs marges, Publ. Inst. Statist. Univ. Paris, 8:229-231, 1959. 